\title{
Optical and structural properties of dislocations in InGaN
}

F.C-P. Massabuau ${ }^{1 *}$, M.K. Horton ${ }^{2}$, E. Pearce ${ }^{1}$, S. Hammersley ${ }^{3,4}$, P. Chen $^{1}$, M.S. Zielinski ${ }^{5}$, T.F.K. Weatherley $^{1}$, G. Divitini ${ }^{1}$, P.R. Edwards ${ }^{6}$, M.J. Kappers ${ }^{1}$, C. McAleese ${ }^{1}$, M.A. Moram ${ }^{7}$, C.J. Humphreys $^{1,8}$, P. Dawson ${ }^{3}$ and R.A. Oliver ${ }^{1}$

${ }^{1}$ Department of Materials Science and Metallurgy, University of Cambridge, Cambridge CB3 OFS, UK

${ }^{2}$ Materials Science and Engineering, University of California Berkeley, Berkeley, CA 94720, US

${ }^{3}$ School of Physics and Astronomy, Photon Science Institute, University of Manchester, Manchester M13 9PL, $U K$

${ }^{4}$ School of Electrical and Electronic Engineering, University of Manchester, Manchester M13 9PL, UK

${ }^{5}$ Attolight AG, EPFL Innovation Park, 1015 Lausanne, Switzerland

${ }^{6}$ Department of Physics, SUPA, University of Strathclyde, Glasgow G4 ONG, UK

${ }^{7}$ Department of Materials, Imperial College London, London SW7 2AZ, UK

${ }^{8}$ School of Engineering and Materials Science, Queen Mary University of London, London E1 4NS, UK

*E-mail: $\underline{\text { m3350@cam.ac.uk }}$

\begin{abstract}
Threading dislocations in thick layers of $\operatorname{In}_{x} \mathrm{Ga}_{1-\mathrm{x}} \mathrm{N}(5 \%<\mathrm{x}<15 \%)$ have been investigated by means of cathodoluminescence, time-resolved cathodoluminescence and molecular dynamics. We show that indium atoms segregate near dislocations in all the samples. This promotes the formation of In-N-In chains and atomic condensates which localize carriers and hinder non-radiative recombination at dislocations. We note however that the dark halo surrounding the dislocations in the cathodoluminescence image becomes increasingly pronounced as the indium fraction of the sample increases. Using transmission electron microscopy, we attribute the dark halo to a region of lower indium content formed below the facet of the V-shaped pit that terminates the dislocation in low composition samples $(\mathrm{x}<12 \%)$. For $\mathrm{x}>12 \%$, the facets of the V-defect featured dislocation bundles instead of the low indium fraction region. In this sample the origin of the dark halo may relate to a compound effect of the dislocation bundles, of a variation of surface potential and perhaps of an increase in carrier diffusion length.
\end{abstract}

\section{Introduction}

Due to a tunable direct bandgap ranging from $0.7 \mathrm{eV}$ to $3.5 \mathrm{eV}, \operatorname{In}_{\mathrm{x}} \mathrm{Ga}_{1-\mathrm{x}} \mathrm{N}$ alloys (hereafter referred to simply as InGaN) find applications in a wide range of optoelectronic devices. In particular, InGaN is of interest for the fabrication of efficient blue and green light emitting diodes (LEDs) [1]. However, this technology, despite being widely used, still suffers from unsolved challenges. One such challenge, 
known as the "green gap" problem, refers to the relatively poor efficiency of devices operating at room temperature in the green part of the spectrum as opposed to those operating in the blue. For example, peak external quantum efficiencies of $86 \%$ and $44 \%$ have recently been reported for blue [2] and green [3] LEDs, respectively. The origin of the green gap is still a matter of debate and several mechanisms to explain it have been suggested [4,5]. In particular, green emission requires the growth of high indium fraction materials at low growth temperature which favors the formation of defects that can act as nonradiative recombination sites or assist the tunneling of carriers from the space charge region into the InGaN active region followed by subsequent non-radiative recombination [6]. There is no agreement in the literature about which defects are to blame for this deleterious phenomenon: dislocations (threading or misfit), trench defects, and point defects have all been linked to a decrease in efficiency in greenemitting samples [7-9].

In an earlier report we showed using cathodoluminescence that threading dislocations in $130 \mathrm{~nm}$ thick InGaN ( $x \sim 9 \%)$ were decorated by indium atoms that segregate in the tensile region of the dislocation [10]. This segregation leads to the enhanced formation of carrier localization centers (In-N-In chains and atomic condensates [11-13]) near the dislocation with respect to the material far away from the dislocation, and can hinder non-radiative recombination at the dislocation core. In the present report, we investigate how the carrier localization properties at dislocations in thick InGaN films may vary with increasing indium content, in the hope of shedding light on the contribution of dislocations to the green gap problem.

\section{Methods}

Four Si-doped InGaN epilayers were grown by metalorganic vapor phase epitaxy (MOVPE) in a Thomas Swan 6×2 inch close-coupled showerhead reactor. Trimethylgallium (TMG), trimethylindium (TMI), diluted silane in hydrogen $\left(\mathrm{SiH}_{4}\right)$ and ammonia $\left(\mathrm{NH}_{3}\right)$ were used as precursors for the $\mathrm{Ga}, \mathrm{In}, \mathrm{Si}$ and $\mathrm{N}$, respectively. Hydrogen $\left(\mathrm{H}_{2}\right)$ was used as the carrier gas for the growth of $\mathrm{GaN}$, while a mixture of hydrogen and nitrogen $\left(\mathrm{N}_{2}\right)$ was employed for $\mathrm{InGaN}$ growth. Pseudo-substrates consisting of $c a .5$ $\mu \mathrm{m}$ of GaN (of which the first $2 \mu \mathrm{m}$ were undoped and the following $3 \mu \mathrm{m}$ were Si-doped at $5 \times 10^{18}$ $\mathrm{cm}^{-3}$ ) grown on $c$-plane sapphire with a miscut of $0.25 \pm 0.10^{\circ}$ towards $(11 \overline{2} 0)$ were employed, yielding a threading dislocation density of approximately $4 \times 10^{8} \mathrm{~cm}^{-2}$. After the growth of a $500 \mathrm{~nm}$ thick GaN layer, the $c a .130 \mathrm{~nm}$ thick $\operatorname{In}_{\mathrm{x}} \mathrm{Ga}_{1-\mathrm{x}} \mathrm{N}$ layers were grown at temperatures of $776^{\circ} \mathrm{C}, 749^{\circ} \mathrm{C}, 710^{\circ} \mathrm{C}$, and $680^{\circ} \mathrm{C}$, leading to an indium fraction of $\mathrm{x}=4.9 \pm 0.5 \%$ (sample A), $8.6 \pm 0.5 \%$ (sample B), $12.4 \pm 0.5 \%$ (sample C), and $14.7 \pm 0.5 \%$ (sample D), respectively, as determined by X-ray diffraction (XRD). The Si-doping of the InGaN layer was approximately $5 \times 10^{18} \mathrm{~cm}^{-3}$. A small flow of $\mathrm{H}_{2}$, i.e. $1-2 \%$ of the total carrier gas flow, was used for the growth of InGaN in order to improve the surface quality [14]. XRD was employed to characterize the composition, thickness and strain state of the films. Using reciprocal space maps on the 006 and 204 reflections we found that the InGaN layer can be considered fully 
strained to the GaN pseudo-substrate - only sample $\mathrm{C}$ and $\mathrm{D}$ exhibit a slight strain relaxation of $5 \pm 2 \%$ and $7 \pm 4 \%$, respectively.
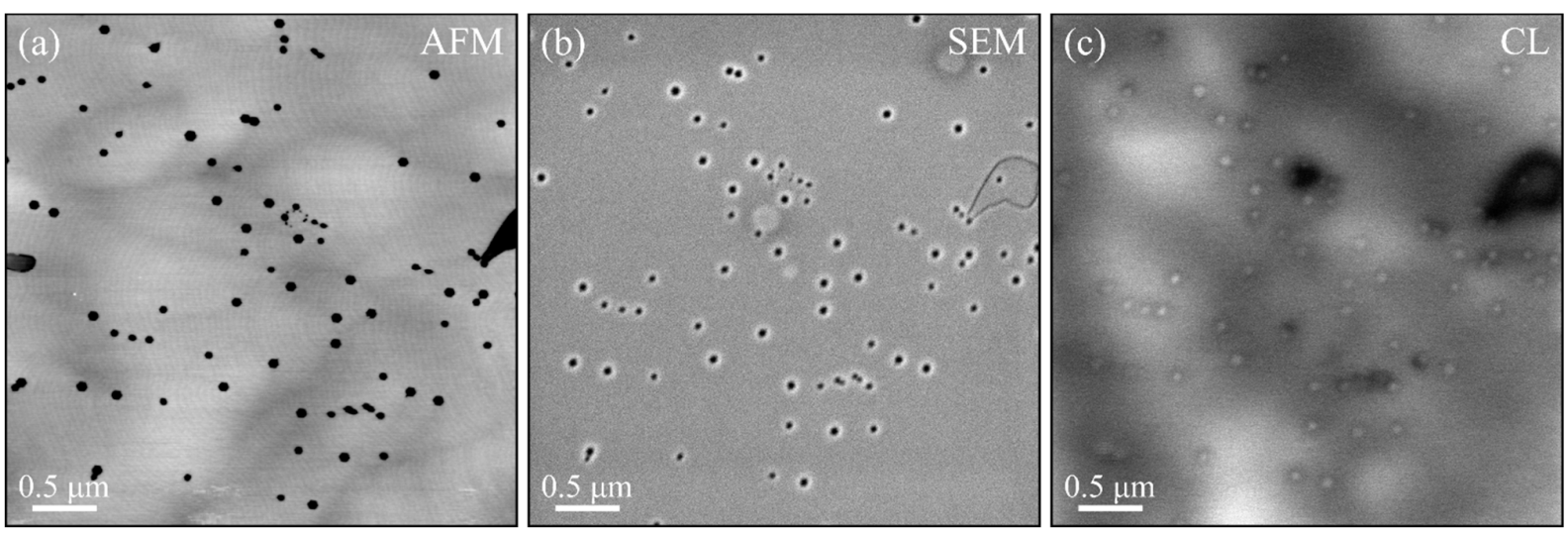

Figure 1 - (a) AFM, (b) SEM, and (c) room temperature panchromatic CL taken on the same area of sample B.

Dislocations were analyzed using a "multi-microscopy" approach (in which the same set of dislocations are investigated under different microscopes) to link the optical properties of each dislocation to its morphological properties. The experimental setup is described in Ref. [15] and employs a copper grid, as is used for transmission electron microscopy (TEM) sample preparation, which is glued on the surface of the sample. Using the $\sim 50 \mu \mathrm{m}$ square mesh of the grid as an initial guide, the same set of dislocations could be identified using several microscopy techniques. Figure 1 shows an example of such an approach, here in the case of sample B, using atomic force microscopy (AFM) and scanning electron microscopy (SEM) combined with cathodoluminescence (CL). AFM was conducted on a Veeco Dimension 3100 operating in tapping mode and was used to characterize the dislocation type (edge, mixed or screw) based on the size of the V-pit that terminates the dislocation [16], as well as the distance to nearest neighbor of each dislocation. SEM-CL was conducted on an FEI XL 30 microscope operated at $3 \mathrm{kV}$ equipped with a Gatan MonoCL4 CL system. Measurements were performed at room temperature $(300 \mathrm{~K})$. Monte Carlo simulations [17] indicate that at $3 \mathrm{kV}, 90 \%$ of the CL signal originates from the first $50 \mathrm{~nm}$ of material below the surface. The slits and diffraction grating of the CL system were set at $0.2 \mathrm{~mm}$ and 1200 lines $/ \mathrm{mm}$, respectively, thus allowing a $0.5 \mathrm{~nm}$ spectral resolution. For sample D, because of the lower intensity of the signal and increased linewidth, these parameters were set at $0.5 \mathrm{~mm}$ and 300 lines $/ \mathrm{mm}$ respectively, resulting in a $5 \mathrm{~nm}$ spectral resolution. The samples exhibited a CL peak emission wavelength at approximately $385 \mathrm{~nm}$ (sample A), $406 \mathrm{~nm}$ (sample B), $426 \mathrm{~nm}$ (sample C), and $465 \mathrm{~nm}$ (sample D), and the light emission was recorded over a $70 \mathrm{~nm}$ (270 $\mathrm{nm}$ for sample D) spectral range around the peak emission. SEM-CL was used to measure the integrated intensity and emission wavelength of various parts of the V-pits (center and facet) relative to the surrounding material. More details on the experimental procedure can be found in Ref. [10]. For each sample, about 500 dislocations were characterized in this manner. 
Metropolis Monte Carlo simulations were performed to obtain statistical information on the expected length and distribution of In-N-In chains in the vicinity of a dislocation core. In an approach similar to that reported elsewhere $[10,18]$, four supercells of dimension $64 \mathrm{a} \times 64 \mathrm{a} \times 16 \mathrm{c}$ containing a random alloy of composition $5.5 \%, 8.6 \%, 12.6 \%$ and $20 \%$ fully strained with regards to $\mathrm{GaN}$ and containing a 5/7atom ring edge type dislocation, were simulated. The number and length of In-N-In chains contained within a cylinder of $1 \mathrm{~nm}$ radius centered on the dislocation core was then recorded, at the initial state (as a representation of a random alloy configuration) and at equilibrium.
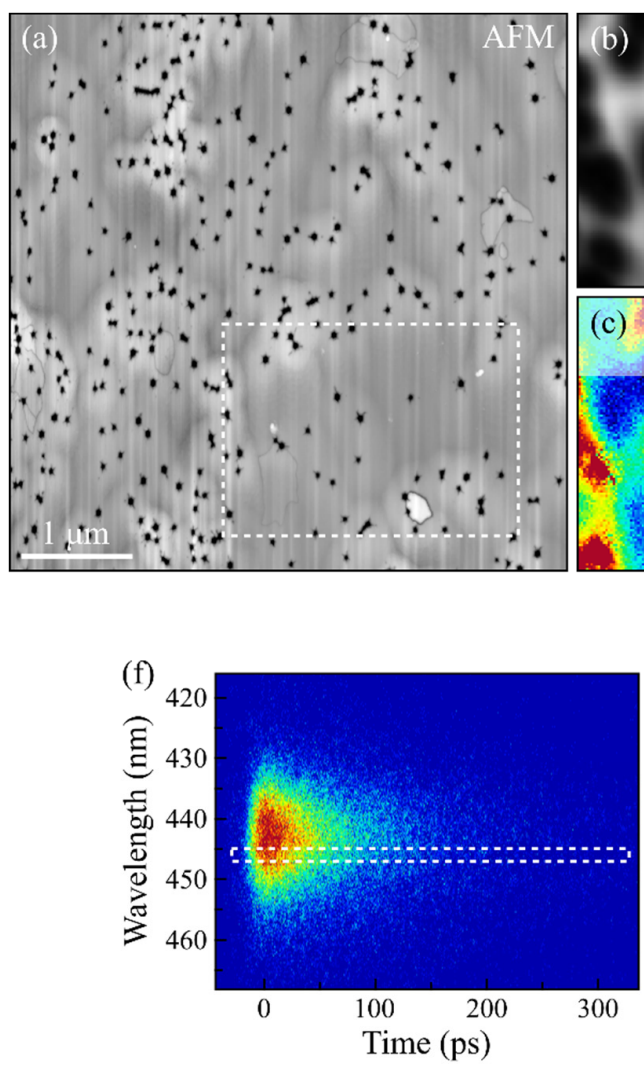
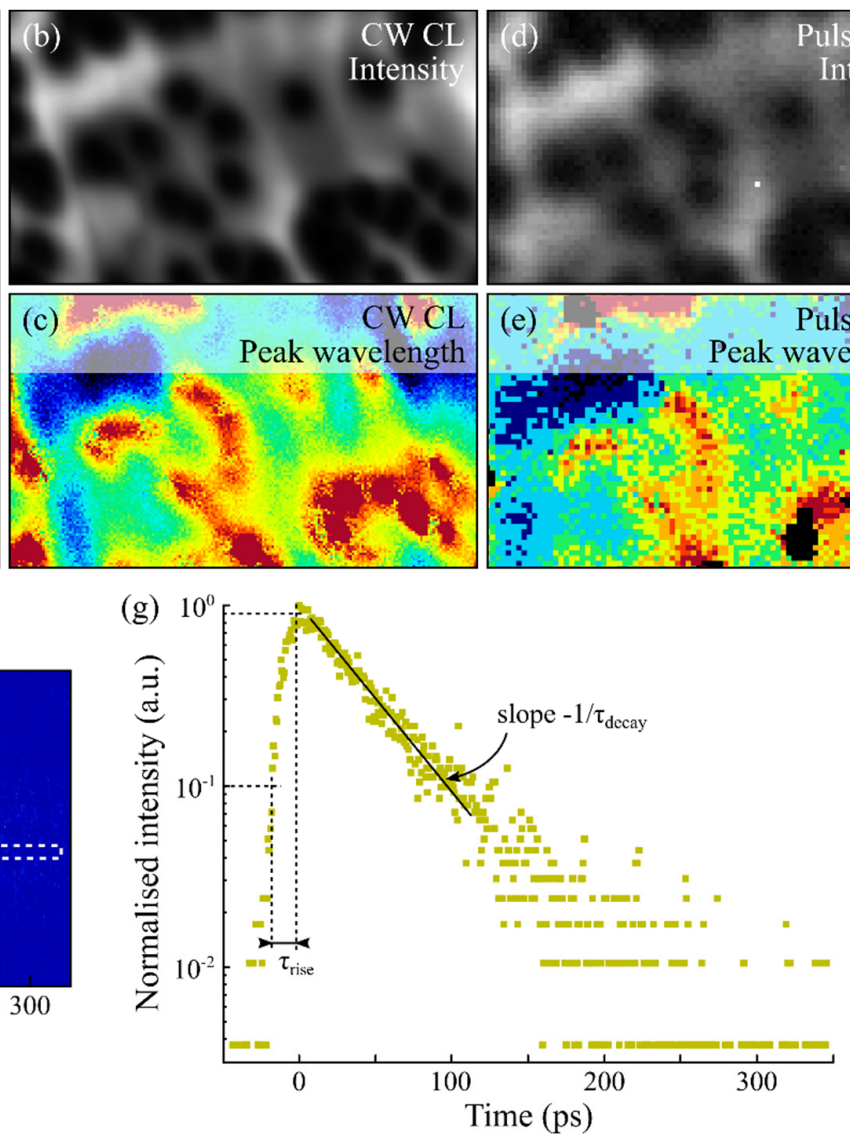

Figure 2 - (a) AFM, continuous wave (CW) mode CL (b) integrated intensity and (c) peak wavelength, and pulsed mode CL (d) integrated intensity and (e) peak wavelength of the same region of sample D at $300 \mathrm{~K}$ (corresponding to the area highlighted in (a)). (f) Streak map taken in the surrounding material, and (g) extracted time decay curve at the spectral position indicated by a square in $(\mathrm{g})$. (The black regions in (e) correspond to pixels where the CL signal was too low to extract accurate value of peak wavelength.)

To understand the carrier dynamics at and around the dislocations, time-resolved CL (TR-CL) measurements were collected using the Attolight Allalin 4027 Chronos SEM-CL microscope. The experimental setup for the TR-CL is composed of three modules: the excitation module, the integrated Attolight SEM-CL module, and the detection module. The excitation module generates ultraviolet light pulses (355 nm, $5 \mathrm{ps}, 80 \mathrm{MHz}$ repetition rate) using a frequency-tripled customized Er:fiber laser. These 
ultraviolet light pulses are coupled with an SEM-CL microscope and irradiate its field emission gun to generate picosecond electron pulses. The Attolight system integrates an achromatic reflective lens (NA=0.71) within the objective lens of the SEM, thus aligning their focal planes and suppressing any necessary alignment. The system integrates a specifically designed cryo-stage allowing for stable and precise measurements at low temperatures ( $\mathrm{drift}<10 \mathrm{~nm} /$ minute). The CL signal is diffracted spectrally by the Czerny-Turner type monochromator (Horiba Scientific, iHR 320) and collected by an Andor Newton 920 CCD camera. For the time-resolved measurements, a streak camera (Optronis SC-10) is used in photon counting mode. For the wavelength used in this experiment, the time response of the overall system is $\sim 12 \mathrm{ps}$. The microscope was operated at $5 \mathrm{kV}$, as a tradeoff between reasonable spatial resolution (using the approach described earlier, we estimate that $90 \%$ of the CL signal here originates from within $\sim 105 \mathrm{~nm}$ below the surface) and signal intensity. Under these conditions, the probe current was $1.6 \mathrm{pA}$. Measurements were performed at 15 and $300 \mathrm{~K}$. Figure 2 shows an example of the CL mapping, with all the data having been obtained on the same area of sample D at $300 \mathrm{~K}$, in continuous wave $(\mathrm{CW})$ mode and pulsed mode. Figure 2(b-e) shows an excellent agreement between the two CL modes (we can resolve individual dislocation in both modes and the peak wavelength remains the same - as evidenced by the common scale bar), and demonstrate our ability to pinpoint a specific location in TR-CL (In this experiment, sample D observed at $300 \mathrm{~K}$ corresponds to the most challenging imaging conditions since this sample under these conditions demonstrates the weakest emission intensity). As illustrated in Figure 2(f), streak maps were recorded inside the bright spot, on the dark halo, and on the material surrounding isolated dislocations which had been identified in the AFM beforehand. For each streak map, the time decay was extracted over a spectral bandwidth of $2 \mathrm{~nm}$ (dotted region in Figure 2(f)). The time decay curves were analyzed to determine the rise time $\tau_{\text {rise }}$, and the decay time $\tau_{\text {decay }}(1 / \mathrm{e}$ lifetime), as shown in Figure $2(\mathrm{~g})$. $\tau_{\text {rise }}$ was obtained as the time between $10 \%$ and $90 \%$ of the peak intensity, and $\tau_{\text {decay }}$ was determined by the slope of the exponential decay curves.

Cross-sectional observation of the samples was conducted by annular dark field scanning TEM (ADFSTEM), diffraction contrast TEM, and energy dispersive X-ray spectroscopy (EDX) in an FEI Tecnai Osiris microscope operated at $200 \mathrm{kV}$. The sample was prepared by standard mechanical polishing, followed by $\mathrm{Ar}^{+}$ion milling at $5 \mathrm{kV}$ and polishing at $1 \mathrm{kV}$ down to $0.1 \mathrm{kV}$. EDX quantification was performed using the Cliff-Lorimer factor method [19].

\section{Results and Discussions}

\section{A. Panchromatic CL}

As can be seen in Figure 1, the position of dislocations, which can be directly identified in the AFM and SEM images as V-pits (inverted hexagonal pyramids that form at the termination of dislocations in In-containing structures [20]), matches the position of a bright spot surrounded by a dark halo in the panchromatic CL image. Additional panchromatic CL images are provided in Figure 3(a-d), showing 
the presence of a bright spot (or region of enhanced CL emission within the dark halo) in all the samples studied here. The presence of the bright spot indicates that there is an enhanced CL signal arising from the center of the V-pit, which appears contrary to current understanding on the behavior of dislocations in semiconductor materials - where dislocations usually act as non-radiative recombination centers and appear only as black spots in the CL [21,22]. However, one cannot rule out the potential impact of the geometry of the V-pit on the CL signal. We therefore used a three-dimensional Monte Carlo simulation (software CASINO 3 [23]) of a GaN film containing an inverted 6-fold pyramid of similar size and depth as the V-pits studied here. The simulation outputs the "absorbed energy", that is, the fraction of the incident beam energy which is not carried away by backscattered electrons and is therefore deposited in the material, and can be reasonably interpreted as carrier density. We predict that at $3 \mathrm{kV}$ (as is used in our panchromatic CL experiment) the carrier density should be about $15-20 \%$ higher in the center of the V-pit than on the surrounding material, and about 5\% lower at the ridge between the inclined facet and horizontal plane (See supplementary material Figure S1). The geometry of the V-pit can therefore play a role in the increased CL signal observed in the center of the V-pit and was consequently taken into account when interpreting our data. Notwithstanding the geometric contribution, we note that the bright spot is indicative of specific material properties. For example, we reported in our earlier study that the bright spot in CL images was rarely centered within the dark halo, which would have been expected were it solely a geometric effect, but instead was often offset in various directions [10]. We also made this observation in the present study, this is particularly visible (circled) in Figure 3(c). Furthermore, we report - in Ref. [10] and later in Figure 3(f) - that the CL emission at the center of the V-pit was redshifted compared to the surrounding material, but such a small variation in carrier density $(<20 \%)$ is not expected to have any measurable effect on the emission wavelength. In that study [10] we therefore concluded that the emission properties of the V-pit center could be attributed to the enhanced formation of In-N-In chains and atomic condensates which results from the segregation of indium atoms in the tensile region of the dislocation. Such features have been shown to act as carrier localization centers [11-13]. 

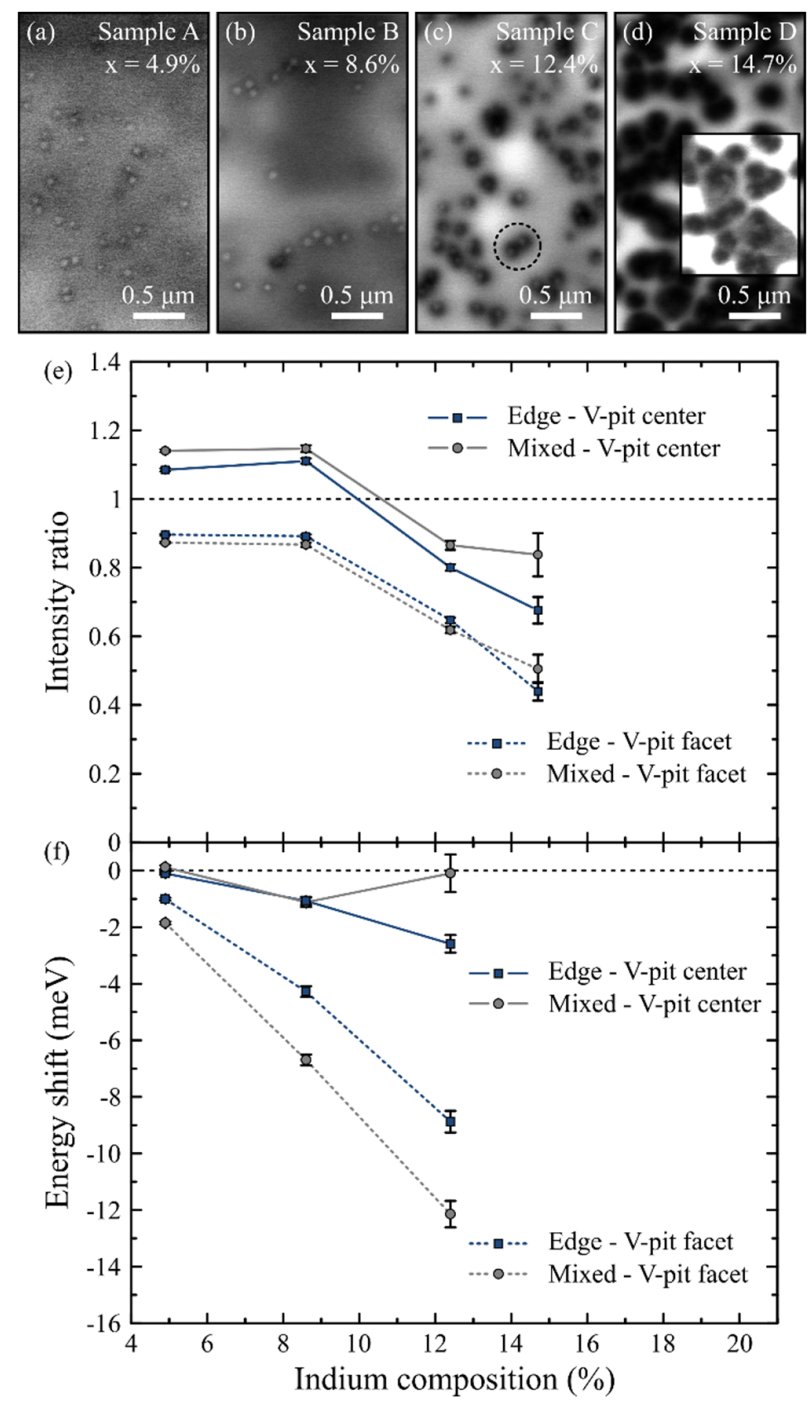

Figure 3 - Room temperature panchromatic CL image of samples (a) A, (b) B, (c) C, and (d) D. In inset of (d), panchromatic $\mathrm{CL}$ with brightness and contrast adjusted to reveal CL intensity variation at dislocations. Plots of the (e) intensity ratio and (f) energy shift on the center and on the facet of the V-pit as a function of indium content and dislocation type.

Figure 3(a-d) shows the $300 \mathrm{~K}$ panchromatic CL images of all four samples investigated. We can see that dislocations in samples A, B and C have a bright spot in the center of the dark halo. While this is not readily noticeable in the dislocations in sample $\mathrm{D}$, a relatively bright spot (or more accurately, a region of enhanced $\mathrm{CL}$ emission within the dark halo) could also be identified by changing the brightness and contrast of the image (inset of Figure 3(d)). Overall, we can see that the bright spots fade away as the composition of indium increases. Another observation is that the dark spot gets noticeably larger with increasing indium content. To quantify the impact of the indium content on the emission, in Figure 3(e) we plot, as a function of indium content, the "intensity ratio" measured by CL, that is, the integrated intensity emitted from the V-pit facet (the term "V-pit facet" is here defined as the position of minimum CL integrated intensity on either side of the bright spot - which coincides with the 
topographical facet of the V-pit although this is less well-defined in high composition samples because of the larger size of the dark halo) or V-pit center relative to that of the surrounding material, and in Figure 3(f) the "energy shift", that is, the peak emission energy emitted from the V-pit facet or V-pit center relative to that of the surrounding material. Please note that since the spectral acquisition range was $70 \mathrm{~nm}$ ( $270 \mathrm{~nm}$ for sample D), only the band-edge emission of the InGaN film is contributing to the intensity ratio measurement here. Also note that the emission signal of Sample D was too low to allow for accurate measurement of the energy shift, which explains the missing data points in Figure 3(f) for this sample. Because we previously observed that the intensity ratio and energy shift vary due to strain effects when two dislocations are too close to each other [10], only the results for isolated dislocations - distance to nearest neighbor $>120 \mathrm{~nm}$ - are included here. The separation above which the presence of an adjacent dislocation did not observably alter the emission properties $(c a .120 \mathrm{~nm})$ did not appear to be noticeably affected by the indium fraction in samples A, B and C (for sample D the spread in values - illustrated by the error bars in Figure 3(e) - is too large to identify a clear cut-off separation, which was therefore taken at $120 \mathrm{~nm}$ for consistency). Figure 3(e-f) present the average intensity ratio and energy shift as a function of the indium content, for different locations on the V-pit (center or facet) and dislocation type (edge and mixed only; the amount of screw-type dislocations being too low to be statistically significant). It can be seen that the intensity ratio (Figure 3(e)) is always higher on the center of the V-pit than on its facets, irrespective of composition or dislocation type. This observation reflects the presence of the bright spot on the panchromatic CL images. We also observed that the bright spot is more intense (intensity ratio $>1$ ) than the surrounding materials for low indium compositions and becomes dimmer (intensity ratio $<1$ ) at higher compositions, in line with our visual inspection of the samples. The fact that the CL emission at the V-pit center is always more intense than on the facet could be influenced by the geometry of the pit; however, the fact that the intensity of the bright spot (or of the dark halo) relative to the surrounding material varies with indium fraction indicates that the properties of the material affect our measurements. Moreover, the fact that samples A and B exhibit an intensity ratio in the V-pit center of 1.10-1.15 is a clear confirmation that an additional mechanism screens non-radiative recombination at dislocation: if the intensity ratio were solely affected by the non-radiative dislocation and the geometric effects (which increases the carrier density by 15 $20 \%$ ) then the intensity ratio should be significantly lower than 1.10-1.15.

Turning our attention to the energy shift (Figure 3(f)), we can see that the emission at the V-pit is almost systematically redshifted compared to the surrounding material, with stronger redshift recorded on the V-pit facet than on the V-pit center. In our earlier study, we attributed the weak redshift of the bright spot to the presence of carrier localization centers (In-N-In chains and atomic condensates) in the vicinity of the dislocation, while the stronger redshift of the dark halo was associated to the strain relaxation that occurs near the V-pit facets [10]. The results presented in Figure 3(f) corroborate this picture. At the center of the V-pit, a small redshift is observed that does not vary significantly with 
indium composition, which may be consistent with the existence of similar localization centers (In-NIn chains and atomic condensates) in all cases. Moreover, we do not see a definite impact of the dislocation type on this measurement. This is not surprising if we consider that the segregation of indium atoms at dislocations (which favors the formation of carrier localization centers) is driven by the presence of a tensile region near the dislocation. A tensile strain region will be present if there is an edge component in the dislocation's Burgers vector (and there will also be a compressive strain region). Given that the screw component only affects the shear strain, it has no expected effect on the tensile and compressive strains, thus edge and mixed type dislocations are expected to behave similarly. On the V-pit facet, the extent of strain relaxation would be expected to vary with composition given that the amount of elastic stress in the material strongly depends on the indium fraction, increasing as the indium fraction is increased. This could explain why the redshift at the facet increases with composition and also why it depends on the dislocation type: the elastic strain at a dislocation is roughly proportional to $\mathrm{Gb}^{2}$ ( $\mathrm{G}$ is the shear modulus and $\mathrm{b}$ is the dislocation Burgers vector), hence the elastic energy of a dislocation with edge character $(\mathbf{b}=\mathbf{a})$ is lower than one with mixed character $(\mathbf{b}=\mathbf{a}+\mathbf{c})$.

\section{B. Molecular Dynamics}
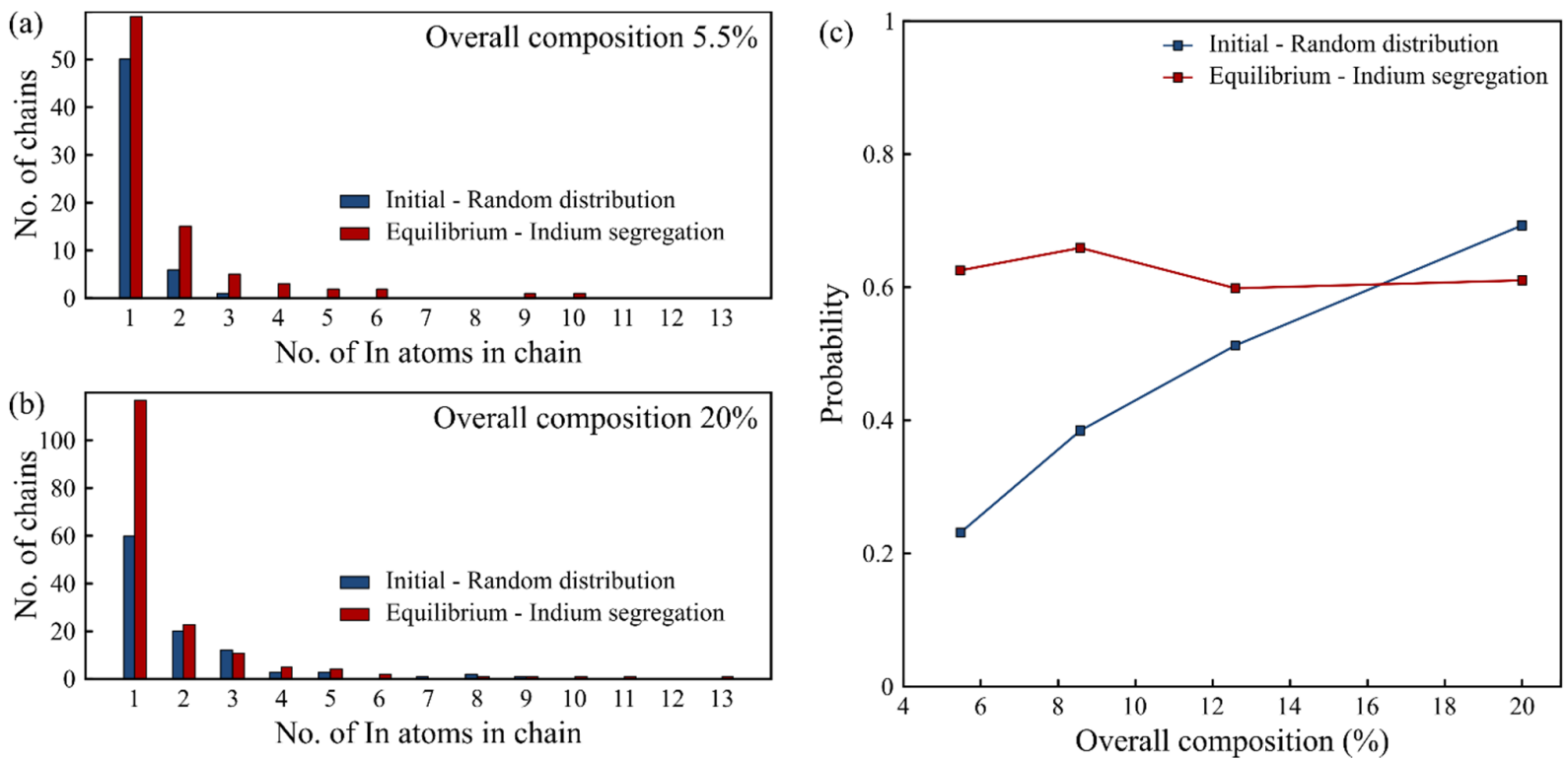

Figure 4 - Molecular dynamics results of distribution and length of In-N-In chains in supercells of overall composition 5.5\% (a) and $20 \%$ (b). (c) Probability of indium atom to be part of multiple atom chain.

A molecular dynamics approach was employed to provide further insights into the composition and configuration of the material at and around the dislocation core. Figure 4(a-b) represent the number and length of In-N-In chains within a $1 \mathrm{~nm}$ radius cylinder centered on a dislocation core in a supercell of overall composition 5.5\% (Figure 4(a)) and 20\% (Figure 4(b)). We notice that at equilibrium both alloys contain a greater total number of indium atoms than in the initial state. We observed this across the four 
simulated cells, thus corroborating our experimental results that indium atoms segregate at the dislocation for all compositions.

We note however a difference in terms of chain configuration between low and high composition alloys. In Figure 4(a) for example, the segregation of indium atoms results in an enhanced formation of chains containing two or more indium atoms. On the other hand, in Figure 4(b), the majority of the additional indium atoms form single atom In-N-In chains (which are then simply In-N chains). Figure 4(c) summarizes this observation for all the simulated alloys, by plotting the probability of an indium atom to be part of a multiple atom chain (i.e. two or more indium atoms). In the random alloy configuration, that is, in the initial state of the computation, we found that the probability increases with composition as could be expected from a statistical point of view. Interestingly in the segregated alloy configuration, that is, at the equilibrium state of the computation, this probability remains constant at $\sim 60 \%$ over the composition range covered here. Let us first consider that the material in the region surrounding a dislocation is a random alloy (in thick $\mathrm{InGaN}$ epilayers the use of $\mathrm{H}_{2}$ in the carrier gas mixture has been reported to improve the homogeneity of CL emission energy across the sample [14], indicating that this is a reasonable first assumption). It can therefore be described by the initial state of the simulation. The simulation results indicate that in a low composition epilayer (e.g. sample A or B), the density of In-NIn chains and atomic condensates (i.e. multiple atom chains) is significantly greater in the close vicinity of the dislocation, where the alloy is segregated, as opposed to the surrounding material, where the alloy is random: Figure 4(a) shows that in the same volume, the increased number of multiple atom In-N-In chains accounts for most of the indium segregation. Conversely, in a high composition epilayer (i.e. sample D) the density of In-N-In chains and atomic condensates remains equivalent in the vicinity of the dislocation and in the surrounding material: Figure 4(b) shows that most of the indium segregation stemmed from an increased number of "single atom In-N chains". Theoretically it has been shown that In-N-In chains involving two or more indium atoms, as opposed to single indium atoms, act as strong carrier localization centers $[12,13]$. Therefore, this tells us that, in low composition samples, the density of localization centers in the very vicinity of the dislocation is greater than in the surrounding material, which supports the presence of the bright spot of equivalent or higher intensity than the surrounding material. On the other hand, in the high composition samples, there is no such contrast in density of localization centers. With no difference in localization between the dislocation core region and the rest of the material, there is no reason why the material at the dislocation core should be less prone to nonradiative recombination, hence leading to the observed weakening of the bright spot. Indeed, with no additional localisation centers in the vicinity of the dislocation core, the existence of non-radiative centers at the dislocation core itself make carriers in this region particularly susceptible to non-radiative recombination.

\section{Time-resolved CL}



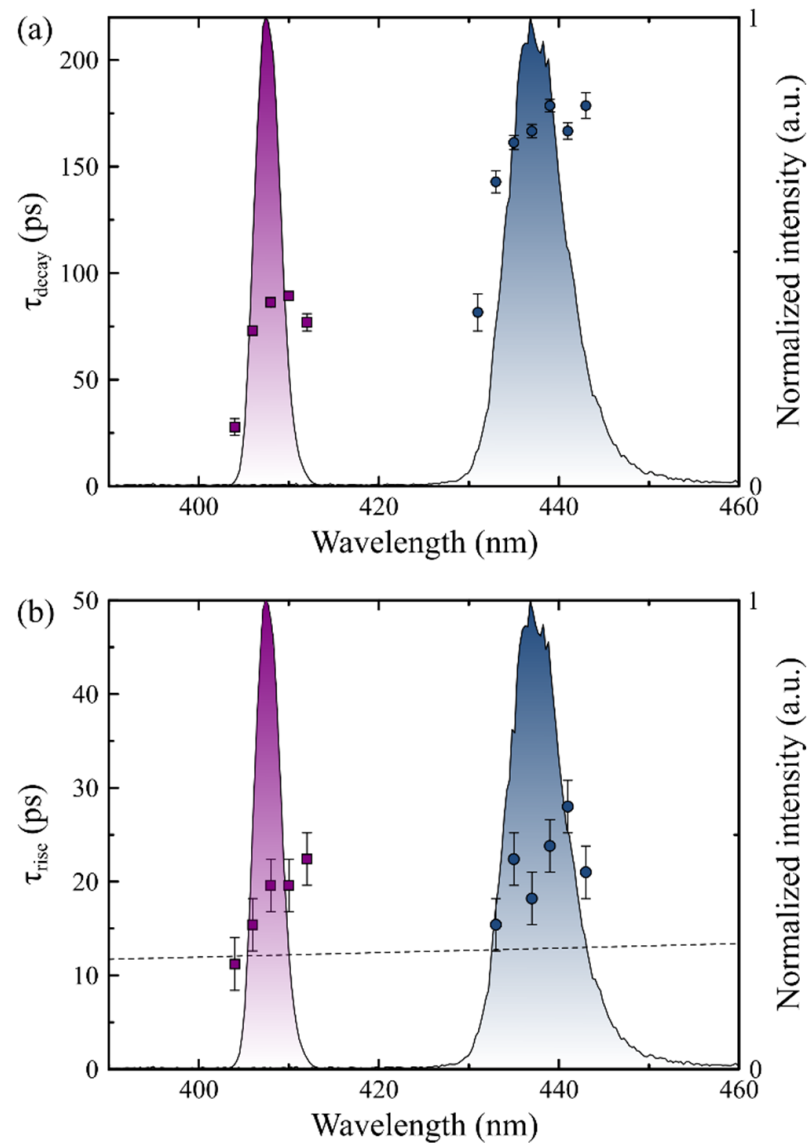

Figure 5 - Wavelength dependence of (a) decay time $\tau_{\text {decay }}$ and (b) rise time $\tau_{\text {rise }}$ recorded at $15 \mathrm{~K}$ on the bright spot of an edge-type dislocation in samples B and D. In background, corresponding time integrated emission spectra (B in violet, and D in blue), and in dotted line, the time response of the system.

In order to gain a better understanding on the carrier dynamics in our samples, TR-CL measurements have been performed on sample B $(x=8.6 \%)$ and sample D $(x=14.7 \%)$. Streak maps have been recorded at $15 \mathrm{~K}$ and $300 \mathrm{~K}$ on regions corresponding to the bright spot, dark spot and surrounding material, and the decay time and rise time were obtained as a function of emission wavelength.

Figure 5(a) shows an example of such analysis conducted at low temperature on both samples, here taken on the bright spot of an edge-type dislocation. (The complete dataset is provided in supplementary material Figures S2-3.) Firstly, we can see that in both samples, the decay time $\tau_{\text {decay }}$ is dependent on the emission wavelength. This point will be discussed later. For now, we will focus on the decay time at the peak wavelength: from Figure 5(a), i.e. on the bright spot, we obtained $\tau_{\text {decay }}$ of $86 \pm 1$ ps and $167 \pm 3$ ps), while in the surrounding material, a decay time $\tau_{\text {decay }}$ at $15 \mathrm{~K}$ of $75 \pm 3$ ps and $166 \pm 2$ ps is measured for sample B and sample D, respectively. Hence, little or no variation of $\tau_{\text {decay }}$ was observed between the bright spot, the dark halo, and the surrounding InGaN material. This result is in contrast with Liu et 
al.'s study, where a similar approach was conducted on threading dislocations in GaN [24]. In that study, conducted at $10 \mathrm{~K}$ and operating voltage of $6 \mathrm{kV}$, $\tau_{\text {decay }}$ was reported to decrease from $125 \mathrm{ps}$ in the surrounding material, to $90 \mathrm{ps}$ at the dislocation (which appeared in the CL images as a black spot, as expected given that no indium was present in the material). In our InGaN study, no such variation was observed, thus indicating that at $15 \mathrm{~K}$ non-radiative recombination near the dislocation is reduced. The carrier density was estimated (from the Monte Carlo simulations - see supplementary material Figure S1) to increase by $15-20 \%$ approximately at the dislocation compared to the surrounding material. We do not expect that a variation in carrier concentration of this magnitude would have a measurable effect on $\tau_{\text {decay }}$. To support this claim, we note that Murotani et al. reported a $9 \%$ increase in $\tau_{\text {decay }}$ (at $5 \mathrm{~K}$ and low excitation regime) for an $\mathrm{InGaN} / \mathrm{GaN}$ quantum wells sample when the optical excitation energy density was increased over about 2 orders of magnitude [25]. Also, Tamulaitis et al. reported on a $300 \mathrm{~K}$ photoluminescence study of a $\mathrm{GaN}$ epilayer that a 10 orders of magnitude increase in excitation power density resulted in a 10 -fold increase in $\tau_{\text {decay }}[26]$.

As mentioned earlier, the decay time is not constant across the emission spectrum. Indeed, we see that both the decay time $\tau_{\text {decay }}$ (Figure 5(a)) and rise time $\tau_{\text {rise }}$ (Figure 5(b)) increase with the emission wavelength at $15 \mathrm{~K}$, irrespective of the probe location (bright spot, dark halo, surrounding). This is indicative of a redistribution of excited carriers in the local potential minima. Indeed, our molecular dynamics results support the idea that the InGaN film (random and segregated configurations alike) will provide an abundance of localization centers within close proximity of where the carriers are first injected (we recall that our In-N-In chain counting was done within a $1 \mathrm{~nm}$ radius cylinder, i.e. a region much smaller than the CL injection volume). Hence, upon generation, carriers experience a large number of potential fluctuations to relax into. Given that these results are reported at low temperature $(15 \mathrm{~K})$ and under low injection regime (probe current $\sim 1.6 \mathrm{pA}$ ), mechanisms such as carrier motion or band filling are unlikely. Instead, carriers most possibly redistribute through a hopping process [27,28]. 

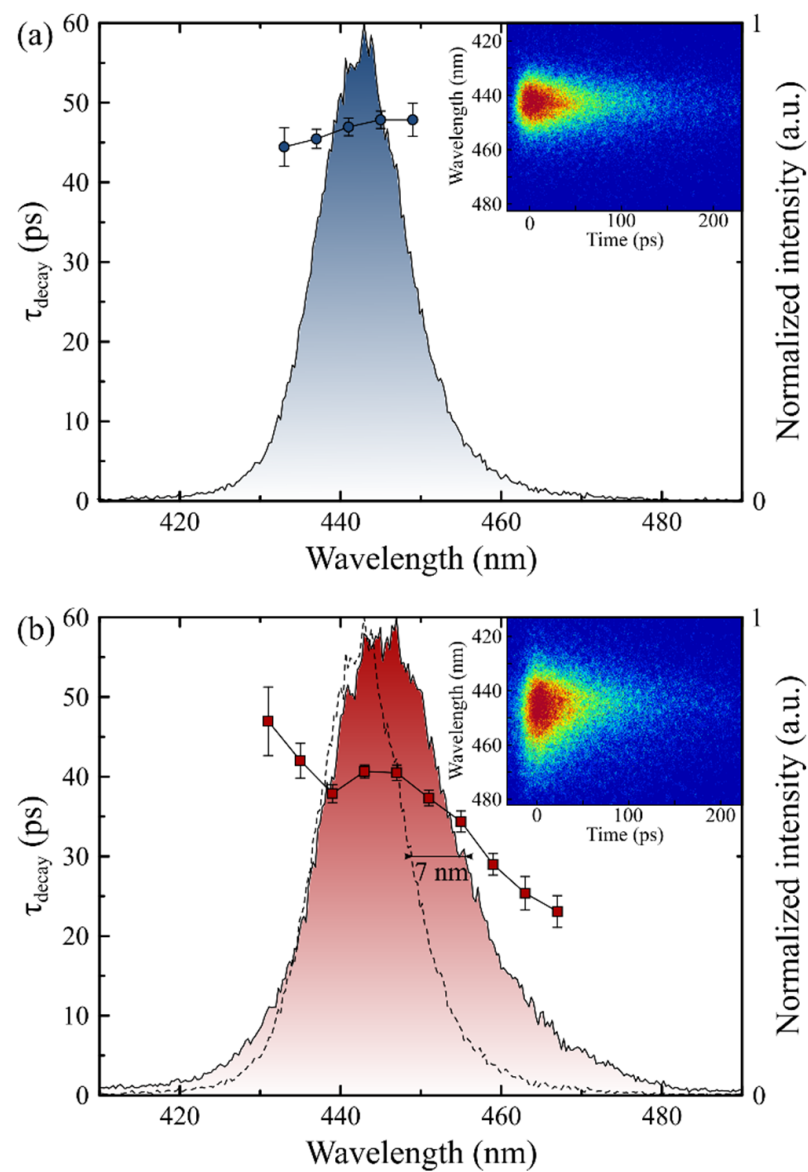

Figure 6 - Wavelength dependence of the decay time $\tau_{\text {decay }}$ recorded at $300 \mathrm{~K}$ on (a) the surrounding material and (b) bright spot of an edge-type dislocation in sample D, with corresponding time integrated emission spectra and streak map. For direct comparison, the surrounding emission spectrum (the same as in (a)) is added as the dotted line in (b).

At room temperature $(300 \mathrm{~K})$, we observed that the $\mathrm{CL}$ spectra broaden and $\tau_{\text {decay }}$ decrease compared to the results obtained at $15 \mathrm{~K}$. The former relates to the thermalization of carriers, while the latter can be ascribed to the increasing effect of Shockley-Read-Hall recombination. Additionally, except for a specific case which will be addressed later on, the decay time $\tau_{\text {decay }}$ becomes almost independent of the detection wavelength, as is illustrated in Figure 6(a). For example, in the surrounding material we report a decay time $\tau_{\text {decay }}$ at $300 \mathrm{~K}$ of $37 \pm 1$ ps and $42 \pm 2$ ps for sample B and sample D, respectively. A wavelength-independent lifetime at high temperature has also been reported using photoluminescence in InGaN quantum wells [29,30], and was attributed to carrier recombination dominated by nonradiative mechanisms.

However, we noticed an exception to this behavior. As illustrated in Figure 6(b), the decay time recorded on the bright spot and dark halo of dislocations was found to decrease for increasing detection wavelength in sample D only. For comparison, Figure 6(a) shows the general behavior, here taken on the surrounding material of sample D. In the bright spot and dark halo (Figure $6(b)), \tau_{\text {decay }}$ can be seen to decrease gradually from $47 \mathrm{ps}$ to $23 \mathrm{ps}$, while in the surrounding material (Figure $6(\mathrm{a})$ ) $\tau_{\text {decay }}$ remains 
at a value near $47 \mathrm{ps}$. The difference between these two behaviors can also be visually perceived from the streak maps shown in insets. We note that, compared to that of the surrounding material, the emission spectrum in the V-pit is broader on the long wavelength side by $7 \mathrm{~nm}$ approximately (measured as indicated in Figure 6(b)). Such spectral shift is of the extent expected from partial strain relaxation of the film [31]. Hence the decrease in $\tau_{\text {decay }}$ with detection wavelength could be attributed to a fast recombination process occurring at the relaxed facets of the V-pit in sample $\mathrm{D}$.

\section{TEM/EDX}

To address this argument, V-pits in sample B and D have been imaged by TEM and are shown in Figure 7. Let us first focus on the ADF-STEM and diffraction contrast TEM data. The data from sample B correspond to the classical picture of a V-pit (Figure 7(a-c)), whereby a dislocation threading towards the surface is terminated by a $\mathrm{V}$-shaped pit. In comparison, sample $\mathrm{D}$ exhibits additional features located on the facets of the V-pit (Figure 7(e-g)). This contrast corresponds to additional dislocations - with $\mathbf{b}=\mathbf{a}$ since they are only visible under $\mathbf{g}=1 \overline{1} 00$ conditions - forming on the facets of the V-pits. These "dislocation bundles" (as they have been called in Ref. [32]) have been seen in the past in thick InGaN layers of similar composition [32-34], and are generated to accommodate the greater strain that is brought by the higher composition of the film [32]. This explains why, despite a similar V-pit density as the other samples, sample D exhibits a greater strain relaxation by XRD. In the context of our TRCL study, this TEM observation reveals that V-pits in sample D (Figure 7(e-h)), as opposed to sample $\mathrm{B}$, are associated with multiple dislocations, resulting in a greater strain relaxation as well as an increase in non-radiative recombination sites. This is consistent with the TR-CL data presented in Figure 6(b) whereby the data recorded on the V-pit center and facet show $7 \mathrm{~nm}$ peak broadening, and a faster time decay on the long wavelength side of the spectrum. We can also notice that the dislocation bundles spread beyond the facets of the V-pit - they seem to affect up to $c a .100 \mathrm{~nm}$ of material on either side of the threading dislocation (Figure 7(e-f)) - which may contribute to explain why the dark halo is larger in sample D than in the other samples. 

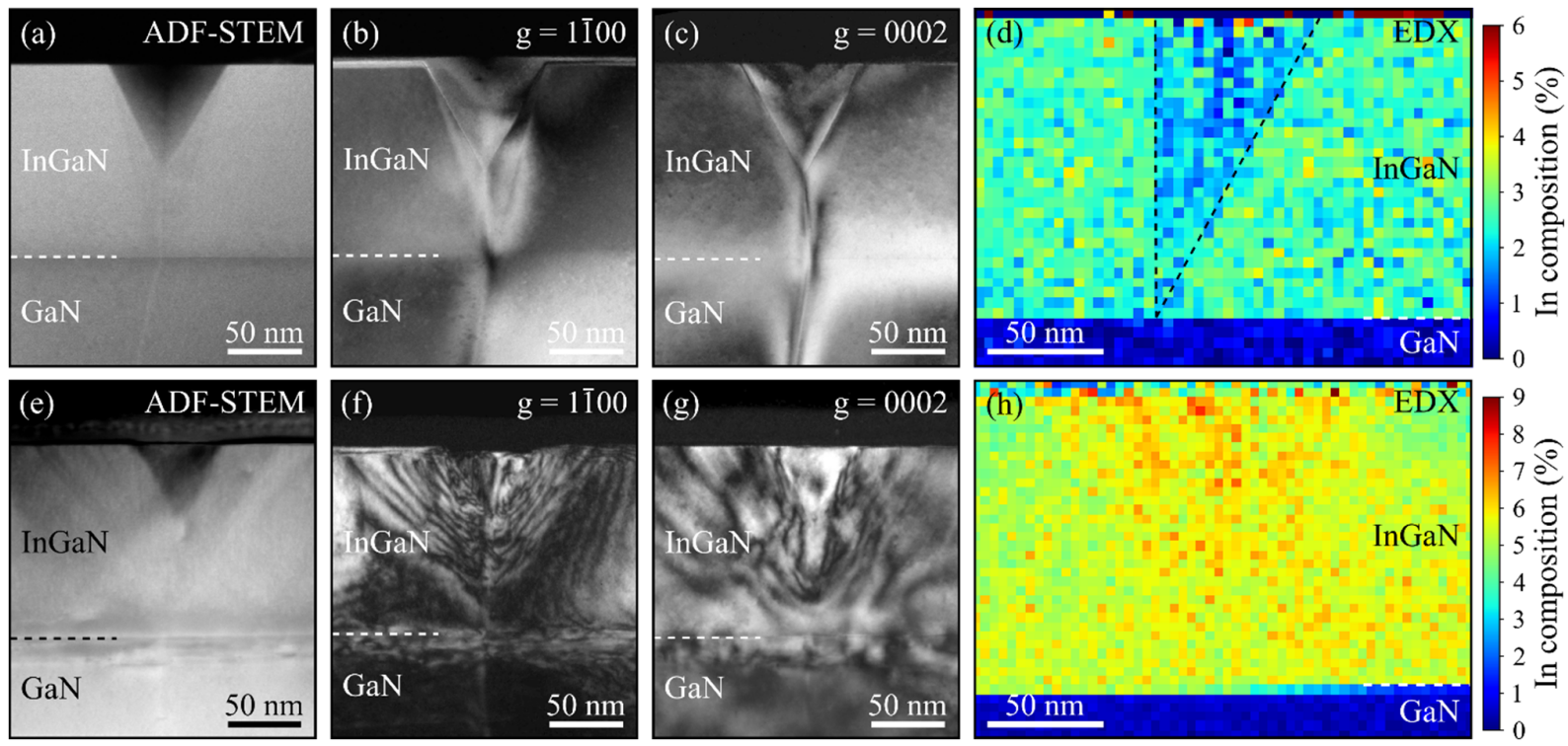

Figure 7 - ADF-STEM (a,e), dark field TEM images taken under $\mathbf{g}=1 \overline{1} 00(\mathrm{~b}, \mathrm{f})$ and $\mathbf{g}=0002(\mathrm{c}, \mathrm{g})$ condition, and EDX mapping of In fraction (d,h) of a V-pit in sample B (a-d) and sample D (e-h).

The composition profile across the V-pits in sample B and sample D have been investigated by EDX, and are shown in Figure 7(d,h). For sample B (Figure 7(d)) we note the presence of a triangular region of lower composition than the surrounding material - the region is bound by a dotted line in the picture. (Note that the asymmetry of the triangle comes from the sample drifting in the TEM.) This feature can also be distinguished in the other pictures (Figure 7(a-c)) and relates to the growth of the V-pit facets. In the context of $\mathrm{V}$-pits formed in quantum well structures, the growth of the sidewall quantum wells on the facets of the V-pit have also been reported to contain less indium [35]. Our EDX results are in line with that study. On the other hand, we can observe that sample D does not share this attribute (Figure 7(h)) - if any, the composition is increased near the V-pit. It is worth pointing out that we did not expect to see (and indeed did not observe) an increase in indium content near the dislocation line because cross-sectional geometry is not well-suited for observing it (plan-view imaging should be preferred in order to avoid issues with projection of areas with different compositions), and also because the atomic segregation is expected to occur at the nanometer scale (as was shown in Ref. [18] for InGaN, or Ref. [36] for AlGaN) which is much smaller than the pixel size of our EDX map.

The EDX data presented in Figure 7 may also help us elucidate, at least partially, the nature of the dark halo that surrounds the bright spot in the CL images. We recall that we observed a dark halo in the CL images taken at $15 \mathrm{~K}$ and $300 \mathrm{~K}$ and that it increases in size with increasing indium fraction (Figure 3(a-d)). Our TR-CL measurements indicate that the dark halo is not associated with a noticeably different decay time, which appears to rule out the possibility that the dark halo originates mainly from non-radiative processes within the dislocation vicinity. The geometry of the V-pit could play a role as the ridges between the surrounding material and the inclined facets provide a convex surface that is 
estimated to reduce the carrier concentration by $c a .5 \%$ - according to our Monte Carlo simulations (See supplementary material Figure S1). This would however not explain the variations in size and intensity across the set of samples.

From the EDX data of sample B (Figure 7(d)), we understand that carriers that are injected in the facets of the V-pits will be injected in a region of lower indium fraction. From the redshifted emission energy of the dark spot it is difficult to conceive that emission occurs from carrier recombination in that lower indium fraction region. Instead carriers will most likely drift to the surrounding material, where the indium content is greater, or will be captured by the localization centers near the dislocation, which would lead to a lower CL emission without affecting noticeably the decay time. This picture could be valid in all the samples containing V-defects with the lower indium fraction facets, and before further strain relaxation mechanism occurs on the facet of the V-pit (i.e. in sample $\mathrm{D}$ ).

In sample $\mathrm{D}$, the presence of additional dislocations on the facets could provide additional non-radiative processes, which is consistent with our $300 \mathrm{~K}$ TR-CL measurements (as discussed before), but conflicts with our $15 \mathrm{~K}$ TR-CL measurements where the decay time was unchanged compared to the surrounding material (See supplementary material Figure S2). A possible explanation would be that the relaxed inclined facets of the V-pit lower the surface potential of the material, which would cause carriers to drift away. Another explanation could be that the diffusion length of carriers increases with indium content, leading to a larger dark halo. This last point would be consistent with the longer decay time measured at $15 \mathrm{~K}$ for sample $\mathrm{D}\left(\tau_{\text {decay }} \sim 165 \mathrm{ps}\right)$ as opposed to sample $\mathrm{B}\left(\tau_{\text {decay }} \sim 80 \mathrm{ps}\right)$.

\section{Conclusion}

In conclusion, we used CL, TR-CL and molecular dynamics to study the optical and structural properties of threading dislocations in thick InGaN layers of composition ranging from 5\% to $15 \%$. Our results indicate that indium atoms segregate in the vicinity of dislocations - within nanometers from the dislocation core - over the range of compositions considered. This favors the formation of In-N-In chains and atomic condensates which localize carriers and impede non-radiative recombination at dislocations. We note however that the dark halo surrounding the dislocation becomes the dominant feature with increasing indium fraction. Whilst for low indium fraction samples $(\mathrm{x}<12 \%)$ we attribute the dark halo to the growth of lower composition material below the facets of the V-pits, for high composition samples $(x>12 \%)$ the origin of the dark halo is less certain, and may be the result of the formation of dislocation bundles on the facets of the V-pits, of a variation of surface potential, and of an increase in carrier diffusion length. We believe that this study will contribute to the elucidation of the role of dislocations to the green gap problem in LEDs, as the mechanisms we report will also be relevant to InGaN quantum wells. However, caution must be exercised because additional effect such as the formation of inclined quantum wells on the $\mathrm{V}$-pit sidewalls and the quantum confined stark effect would further complicate the picture. 


\section{Supplementary Material}

Figure S1- Monte Carlo simulations showing the absorbed energy profile across a V-pit.

Figure S2 and S3 - Wavelength dependence of decay time $\tau_{\text {decay }}$ recorded at $15 \mathrm{~K}$ (Figure S2) and 300 $\mathrm{K}$ (Figure S3) on the bright spot, the dark halo, and the surroundings of an edge-type dislocation in samples B and D.

\section{Acknowledgements}

This project is funded by the European Research Council under the European Community's Seventh Framework Programme (FP7/2007-2013)/ERC grant agreement No. 279361 (MACONS). M.M. acknowledges financial support from the ERC Starting Grant 307636 "SCOPE". M.H. would like to acknowledge support from the Lindemann Trust Fellowship. PD and SH acknowledge the support of the United Kingdom Engineering and Physical Sciences Research Council under grant EP/M010627/1.

\section{References}

[1] S. Nakamura, Ann. Phys. 527, 335 (2015)

[2] Y. Narukawa, M. Ichikawa, D. Sanga, M. Sano, and T. Mukai, J. Phys. D: Appl. Phys. 43, $354002(2010)$

[3] A.I. Alhassan, R.M. Farrell, B. Saifaddin, A. Mughal, F. Wu, S.P. Denbaars, S. Nakamura, and J.S. Speck, Opt. Express 24, 17868 (2016)

[4] M.H. Crawford. IEEE J. Sel. Top. Quantum Electron. 15, 1028 (2009)

[5] B. Ding, Materials Science and Technology 34, 1615 (2018)

[6] N.I. Bochkareva and Y.G. Shreter, Semiconductor 52, 934 (2018)

[7] T. Langer, H. Jonen, A. Kruse, H. Bremers, U. Rossow, and A. Hangleiter, Appl. Phys. Lett. 103, 022108 (2013)

[8] F.C-P. Massabuau, M.J. Davies, F. Oehler, S.K. Pamenter, E.J. Thrush, M. J. Kappers, A. Kovacs, T. Williams, M.A. Hopkins, C.J. Humphreys, P. Dawson, R.E. Dunin-Borkowski, J. Etheridge, D.W.E. Allsopp, and R.A. Oliver, Appl. Phys. Lett. 105, 112110 (2014)

[9] S. Hammersley, M.J. Kappers, F.C-P. Massabuau, S.-L. Sahonta, P. Dawson, R.A. Oliver, and C.J. Humphreys, Appl. Phys. Lett. 107, 132106 (2015)

[10] F.C-P. Massabuau, P. Chen, M.K. Horton, S.L. Rhode, C.X. Ren, T.J. O'Hanlon, A. Kovacs, M.J. Kappers, C.J. Humphreys, R.E. Dunin-Borkowski, and R.A. Oliver, J. Appl. Phys. 121, 013104 (2017)

[11] S. Chichibu, A. Uedono, T. Onuma, B. Haskell, A. Chakraborty, T. Koyama, P. Fini, S. Keller, S. Denbaars, J. Speck, U. Mishra, S. Nakamura, S. Yamaguchi, S. Kamiyama, H. Amano, I. Akasaki, J. Han, and T. Sota, Nat. Mater. 5, 810 (2006) 
[12] Q. Liu, J. Lu, Z. Gao, L. Lai, R. Qin, H. Li, J. Zhou, and G. Li, Phys. Status Solidi B 247, 109 (2010)

[13] S. Schulz, O. Marquardt, C. Coughlan, M. Caro, O. Brandt, and E. O’Reilly, Proc. SPIE 9357, 93570C (2015)

[14] E. Taylor, F. Fang, F. Oehler, P. Edwards, M. Kappers, K. Lorenz, E. Alves, C. McAleese, C. Humphreys, and R. Martin, Semicond. Sci. Technol. 28, 065011 (2013)

[15] F. Massabuau, L. Trinh-Xuan, D. Lodie, E. Thrush, D. Zhu, F. Oehler, T. Zhu, M. Kappers, C. Humphreys, and R. Oliver, J. Appl. Phys. 113, 073505 (2013)

[16] R. Oliver, M. Kappers, J. Sumner, R. Datta, and C. Humphreys, J. Cryst. Growth 289, 506 (2006)

[17] D. Drouin, P. Hovington, and R. Gauvin, Scanning 19, 20 (1997)

[18] M. Horton, S. Rhode, S.-L. Sahonta, M. Kappers, S. Haigh, T. Pennycook, C. Humphreys, R. Dusane, and M. Moram, Nano Lett. 15, 923 (2015)

[19] G. Cliff, and G.W. Lorimer, J. Microsc. 103, 203 (1975)

[20] N. Sharma, P. Thomas, D. Tricker, and C. Humphreys, Appl. Phys. Lett. 77, 1274 (2000)

[21] T. Sugahara, H. Sato, M. Hao, Y. Naoi, S. Kurai, S. Tottori, K. Yamashita, K. Nishino, L.T. Romano, and S. Sakai, Jap. J. Appl. Phys. 37, 398 (1998)

[22] D. Cherns, S.J. Henlay, and F.A. Ponce, Appl. Phys. Lett. 78, 2691 (2001)

[23] H. Demers, N. Poirier-Demers, A.R. Couture, D. Joly, M. Guilmain, N. De Jonge, and D. Drouin, Scanning 33, 135 (2011)

[24] W. Liu, J.-F. Carlin, N. Grandjean, B. Deveaud, and G. Jacopin, Appl. Phys. Lett. 109, 042101 (2016)

[25] H. Murotani, Y. Yamada, Y. Honda, and H. Amano, Phys. Status Solidi B 252, 940 (2015)

[26] G. Tamulaitis, J. Mickevicius, P. Vitta, A. Zukauskas, M.S. Shur, Q. Fareed, and R. Gaska, Superlattice. Microst. 40, 274 (2006)

[27] G. Jacopin, M. Shahmohammadi, J.-D. Ganiere, and B. Deveaud, Appl. Phys. Lett. 104, 042109 (2014)

[28] M. Shahmohammadi, J.-D. Ganière, H. Zhang, R. Ciechonski, G. Vescovi, O. Kryliouk, M. Tchernycheva, and G. Jacopin, Nano Lett. 16, 243 (2016)

[29] Y.H. Cho, G. H. Gainer, A. J. Fischer, J. J. Song, S. Keller, U. K. Mishra, and S. P. DenBaars, Appl. Phys. Lett. 73, 1370 (1998)

[30] M. Gladysiewicz, R. Kudrawiec, M. Syperek, J. Misiewicz, M. Siekacz, G. Cywinski, A. Khachapuridze, T. Suski, C. Skierbiszewski, Appl. Phys. A 115, 1015 (2014)

[31] G. Orsal, Y. El Gmili, N. Fressengeas, J. Streque, R. Djerboub, T. Moudakir, S. Sundaram, A. Ougazzaden, and J. Salvestrini, Opt. Mater. Express 4, 1030 (2014) 
[32] C. Bazioti, E. Papadomanolaki, Th. Kehagias, T. Walther, J. Smalc-Koziorowska, E. Pavlidou, Ph. Komninou, Th. Karakostas, E. Iliopoulos, and G. P. Dimitrakopulos, J. Appl. Phys. 118, 155301 (2015)

[33] F.A. Ponce, S. Srinivasan, A. Bell, L. Geng, R. Liu, M. Stevens, J. Cai, H. Omiya, H. Marui, and S. Tanaka, Phys. Status Solidi B 2, 273 (2003)

[34] S.L. Rhode, W.Y. Fu, M.A. Moram, F.C.-P. Massabuau, M.J. Kappers, C. McAleese, F. Oehler, C.J. Humphreys, R.O. Dusane, and S.-L. Sahonta, J. Appl. Phys. 116, 103513 (2014)

[35] S. Tomiya, Y. Kanitani, S. Tanaka, T. Ohkubo, and K. Hono, Appl. Phys. Lett. 98, 181904 (2011)

[36] F.C-P. Massabuau, S.L. Rhode, M.K. Horton, T.J. O’Hanlon, A. Kovacs, M.S. Zielinski, M.J. Kappers, R.E. Dunin-Borkowski, C.J. Humphreys, and R.A. Oliver, Nano Lett. 17, 4846 (2017) 